gen. A dose-dependent increase in new bone formation was observed over a pe riod of 2-6 weeks in the largest defects re ceiving the GAM, but not in controls. Results for the smaller defects were qualitatively consistent with this general trend although different in rough quantitative terms. Histological analysis of the regenerated tissue morphology and immunohistochemical determination of osteoclast presence likewise showed substantial healing at the higher DNA doses, in contrast to its absence at the lower doses. Expression of plasmid-derived mRNA and protein was reported for 2-3 weeks after implant, even for the lowest levels of plasmid, although not in sufficient amounts to indicate correlation with DNA dose.

These important findings need to be followed by continuing and broader studies, including a more quantitative assessment of the factors that govern the success of such implants. For example, more information is needed about methods to improve plasmid availability, uptake and expression by various cell types. Further analysis of the protein concentrations and distribution range required to achieve a therapeutic effect should help us to better interpret the current tissue regeneration outcomes and to improve fundamental design principles.

Oneinteresting observation reported by Bonadio, et al. is that low doses of administered plasmid did not lead to bone re generation, whereas higher doses did, even though both high and low plasmid concentrations result in transgene expression by $30-50 \%$ of the cells in the wound bed granulation tissue. Another interesting and related question is why plasmid DNA entrapped within a matrix works more effectively than plasmid directly injected into a wound site simply as naked DNA (ref. 8). Finally, there is the issue of selectivity of cell uptake and expression. The wound bed attracts macrophages, fibroblasts and endothelial cells along with tissue-specific cell types such as osteoclasts and osteoblasts or precursors, so the effect of the transgene-encoded therapeutic protein could be strongly influenced by the cell type that takes it up and expresses it-important not only for the degree of benefit but also for toxicity. Developing strategies to target specific cell types through receptor-ligand interactions could enhance GAM performance by providing better control of protein expression and function. Such techniques, however, will involve further, more com- plex design issues?.

Overall, the findings by Bonadio et al. auger considerable promise for combining gene therapy and tissue engineering methodologies to enhance tissue regeneration. Similar multi-disciplinary efforts combining molecular biology, cell biology, biochemistry and bioengineering will lead to substantial advances in this technology.

1. Bonadio, J., E. Smiley, P. Patil, \& Goldstein, S Localized, direct plasmid gene delivery in vivo: prolonged therapy results in reproducible tissue regeneration. Nature Med. 5, 753-759 (1999).

2. Shea, L.D., Smiley, E., Bonadio, J. \& Mooney, D.J DNA delivery from polymer matrices for tissue engineering. Nature Biotechnol. 17, 551-554 (1999).

3. Barr, E. \& Leiden, J. Systemic delivery of recombinant proteins by genetically modified myoblasts. Science 254, 1507-1512 (1991).

4. Langer, R. \& Vacanti, J.P. Tissue engineering Science 260, 920-926 (1993).

5. Eming, S.A., M.L. Yarmush, \& Morgan, J.R. Enhanced function of cultured epithelium by genetic modification: cell-based synthesis and deliv-

ery of growth factors. Biotechnol. Bioeng. 52, 15-23 (1996).

6. Legendre, J.-Y. \& Szoka, F.C.Jr. Delivery of plasmid DNA into mammalian cell lines using $\mathrm{pH}$-sensitive liposomes: comparison with cationic liposomes. Pharmacol. Res. 9, 1235-1242 (1992).

7. Petersen, M.J. et al. Sustained production of human transferrin by transduced fibroblasts implanted into athymic mice: a model for somatic gene therapy. J. Invest. Dermatol. 104, 171-176 (1995).

8. Truong-Le, V.L., August, J.T. \& Leong, K.W. Controlled gene delivery by DNA-gelatin nanospheres. Hum. Gene Ther. 9, 1709-1717 (1998).

9. Schaffer, D.V. \& Lauffenburger, D.A. (1998). Optimization of cell surface binding enhances efficiency and specificity of molecular conjugate gene delivery. J. Biol. Chem. 273, 28004-28009 (1998).

${ }^{1}$ Biotechnology Process Engineering Center and Division of Bioengineering \&

Environmental Health,

Massachusetts Institute of Technology

Cambridge, Massachusetts 02139, USA

${ }^{2}$ Department of Chemical Engineering,

University of California at Berkeley

Berkeley, California, 94720, USA

\title{
Two lesions are better than one for spinal cord regeneration
}

Whereas it once seemed a matter of biological fact that neurons of the central nervous system (CNS) do not regrow after being severed, advances in neuroscience research have made considerable progress in turning this dogma on its head. Another piece of evidence to contradict the doctrine that CNS lesions are irreversible was provided recently by Simona Neuman and Clifford Woolf (Neuron 23, 83-91; 1999), who found that sectioning a peripheral nerve before its central transection can actually stimulate regrowth of the neuronal fibers in the CNS.

Axonal growth into the dorsal column of the CNS stops typically at the site of injury (arrows) when a spinal lesion occurs (upper panel). However, Neuman and Woolf made preconditioning lesions by peripheral sciatic nerve transection 1 or 2 weeks before or 2 weeks after bilateral dorsal column lesions of rat spinal cords (T6-T7).

Anterograde neuronal tracing by injection of conjugated horseradish peroxidase showed that preconditioning results in growth of the ipsilateral sciatic central axons into and across the severed spinal cord (pink fibers, lower panel). The most extensive fiber regrowth was seen with preconditioning at 1 week before central transection. No re-

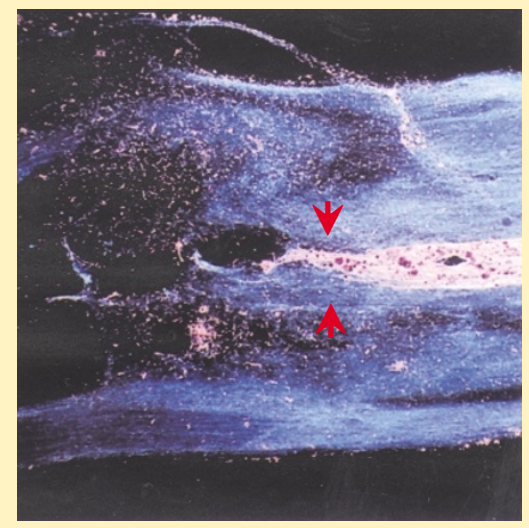
growth was found when peripheral lesions were made after central transection.

The findings reinforce the idea that inhibition of CNS regrowth depends at least partly on the internal state of the growing axons, rather than solely on the neuronal environment. If the way in which preconditioning lesions alter the instrinsic growth state of CNS neurons can be understood and therapeutically harnessed, this basic research could offer clinical hope to paralyzed individuals.

Karen Birmingham 